\title{
The 21-Year Trend of Stroke Incidence in a General Japanese Population: Results from the Takashima Stroke Registry, 1990-2010
}

\author{
Naoyuki Takashima ${ }^{a, b}$ Hisatomi Arimac Tanvir Chowdhury Turin ${ }^{\mathrm{c}}$ \\ Yasuyuki Nakamuraa, e,f Hideki Sugiharag Yutaka Moritah Akira Okayamaa, e, i \\ Katsuyuki Miuraa, e Hirotsugu Ueshimaa, e Yoshikuni Kita ${ }^{a, j}$ \\ on behalf of the Takashima Study Group
}

\begin{abstract}
aDepartment of Public Health, Shiga University of Medical Science, Otsu, Japan; ${ }^{\mathrm{b}}$ Department of Public Health, Kindai University Faculty of Medicine, Osaka-Sayama, Osaka, Japan; 'Department of Preventive Medicine and Public Health, Faculty of Medicine, Fukuoka University, Fukuoka, Japan; dDepartment of Family Medicine, University of Calgary, Calgary, AB, Canada; 'NCD Epidemiologic Research Center, Shiga University of Medical Science, Otsu, Japan; ${ }^{f}$ Medical Examination Center, Yamashina Racto Clinic and Medical Examination Center, Kyoto, Japan;

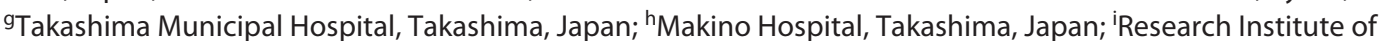
Strategy for Prevention, Tokyo, Japan; 'Tsuruga Nursing University, Tsuruga, Fukui, Japan
\end{abstract}

\section{Keywords}

Stroke $\cdot$ Trend $\cdot$ Incidence $\cdot$ General population

\begin{abstract}
Introduction: Since the 1960s, the stroke morality rate in Japan has declined significantly. Although several risk factors for stroke have become more evident due to increasingly Westernized lifestyle, there have been no population-based registry data on recent time trends in stroke incidence in Japan. The purpose of this study is to determine these trends in stroke incidence using a population-based registry. Methods: Data were obtained from the Takashima Stroke Registry, which covers approximately 50,000 residents in Takashima City, Japan. The age- and sex-standardized stroke incidence rate was estimated using the direct method. Average annual relative changes of stroke incidence were estimated using Poisson regression models. Results: We identified a total of 2,371 patients with first-ever stroke during the period 1990-2010. Crude incidence rates of total stroke (per 100,000
\end{abstract}

person-years) were 225 in the early period (1990-2001) and 187 in the late period (2002-2010), and the mean age at onset of stroke increased from 71.9 years in the early period to 74.8 years in the late period. Age- and sex-standardized incidence rates of stroke (per 100,000 person-years) decreased from 327 in the early part of this study period and 206 in the later period. During the 21-year period, age- and sex-standardized average annual relative reduction in stroke incidence was statistically significant $(-3.7 \%$ per year). When stratified into early and late periods, the adjusted annual relative reduction in stroke incidence was observed during the early period $(-2.1 \%$ per year), and there were no significant changes during the late period. Conclusions: In this population-based registry, there was a significant reduction in incidence of stroke from 1990 to 2010. Although the speed of reduction in stroke incidence appears to have slowed down after 2000, continuous public health measures are required to provide further protection against stroke.

(c) 2022 The Author(s).

Published by S. Karger AG, Basel karger@karger.com www.karger.com/ced

Karger ${ }^{\prime \prime} \div$

BOPEN ACCESS
(C) 2022 The Author(s)

Published by S. Karger AG, Basel

This is an Open Access article licensed under the Creative Common Attribution-NonCommercial-4.0 International License (CC BY-NC) (http://www.karger.com/Services/OpenAccessLicense), applicable to the online version of the article only. Usage and distribution for commercial purposes requires written permission.
Correspondence to:

Naoyuki Takashima,n.takashima@med.kindai.ac.jp 


\section{Introduction}

Stroke is one of the leading causes of premature death and disability in Japan as well as in other countries globally [1]. In Japan, the age-standardized stroke morality rates declined dramatically from the 1960 s to the 1990s [2, $3]$, and this decreasing trend slowed down, but the agestandardized stroke morality rate continued to decrease from 1990s to 2010s [4]. There were few studies to report the trend of stoke incidence in Japan. The age-adjusted stroke incidence rates dramatically decreased from the 1960s and the 1970s, but this trend has been reported to slow down from the 1970s to 2000s [5]. In Japan, smoking and hypertension are both major risk factors for stroke, and both smoking rate and mean systolic blood pressure (BP) have been decreasing over the last several decades [3, $6,7]$. The reduction in the incidence and mortality of stroke in Japan may be attributable to decrease in population BP levels and in smoking rates [3, 7]. In contrast, with the Westernized lifestyle changes, serum cholesterol levels $[3,7]$ and prevalence of diabetes [8], which are also important risk factors for stroke, have increased over the last five decades. However, recent time trends in stroke incidence in general Japanese population are scant in Japan. The aim of the present study is to investigate the 21-year trend in first-ever incidence of stroke and its subtypes using data from the Takashima Stroke Registry $[9,10]$.

\section{Methods}

\section{Study Area and Study Design}

The Takashima Stroke Registry is a part of the Takashima Cardiocerebrovascular Disease Registration Study established in 1989 in Takashima City, Shiga Prefecture, Japan [9-11]; its design has been described elsewhere in detail $[9,10]$. In brief, the Takashima Stroke Registry is an ongoing population-based registry study designed to build a complete information system regarding all cases of acute ischemic and nontraumatic hemorrhagic stroke among residents of Takashima City. Our registry system covered all the hospitals in Takashima City. More than $98 \%$ of hospital admissions of Takashima City were seen in these hospitals [9]. We collocated information of all stroke events from not only inpatient's medical records but also from emergency room records (for death in emergency rooms and death on/during arrival at the hospital).

Takashima City is in the northern part of the Shiga Prefecture. In 2005, as the result of a municipal merger, Takashima County was converted into Takashima City. The population of Takashima County was 52,009 (25,562 men and 26,447 women) in the 1990 census and that of Takashima City in the 2010 census was 52,440 (25,530 men and 26,910 women). The proportion of aging (i.e., aged 65 years and older) was $16.5 \%$ in 1990 and $27.9 \%$ in 2010 . The Takashima Stroke Registry was approved by the Institutional Review Board of the Shiga University of Medical Science (R2000-014).

\section{Medical Histories}

Information on medical history, including transient ischemic attack, use of BP-lowering, glucose-lowering, and lipid-lowering therapies, and smoking and drinking status before onset of the index stroke was obtained from the medical records. Smoking and drinking habits were categorized as never, past, and current.

Stroke was defined as the sudden onset of focal neurological deficits persisting for more than $24 \mathrm{~h}$ according to the Monitoring of Trends and Determinants in Cardiovascular Disease (WHOMONICA) projects [12]. In this analysis, first-ever stroke was defined as the first occurrence of any type of stroke. First-ever strokes were classified as ischemic stroke, intracerebral hemorrhage, subarachnoid hemorrhage, and undetermined type.

\section{Statistical Analysis}

Incidence rates of stroke and its subtypes were calculated using a person-year approach (i.e., total number of stroke cases/sum of the total number of the city population on October 1 of each year [based on the census data] throughout the observation period) separately for the early period (1990-2001) and late period (20022010). The incidence rates were standardized for age and sex using the direct method with the total population of Japan in 2015 [4] as the standard.

The average annual relative changes in stroke incidence across the total study period (1990-2010) were examined using Poisson regression models. Stroke incidence for each year was modeled as a Poisson distribution. The Poisson regression procedure was fitted to the following model: "log (number of incidence cases) = $\alpha+\beta$ (year)." The population number was used as offset in our models. The average annual relative changes in incidence and their 95\% confidence intervals (CIs) were calculated using the regression coefficient of the explanatory variable "year" $(\beta)$ and its standard error as "exp $(\beta \pm 1.96 \times$ standard error)." We also calculated age- and sex-standardized average annual relative change using age- and sex-standardized Poisson regression models. As a sensitivity analysis, we additionally calculated the average annual relative change in incidence separately for the early period (1990-2001) and the late period (2002-2010). A $p$ value of less than 0.05 was considered statistically significant. All analyses were performed using SAS 9.4 (SAS Institute, Cary, NC, USA).

\section{Results}

A total of 2371 first-ever stroke cases were identified from January 1, 1990, to December 31, 2010 (crude incidence rate 209 per 100,000 person-years): 1,464 cases (225 per 100,000 person-years) in the early period (19902001 ) and 907 cases ( 187 per 100,000 person-years) in the late period (2002-2010). Table 1 shows the characteristics of first-ever stroke patients. The mean age at onset of stroke increased from 71.9 years in the early period to 74.8 years in the late period. In the late period, frequency of patients who received BP-lowering medications before onset was significantly higher than that in the early peri- 
Table 1. Characteristics of first-ever stroke patients in early (1990-2001) and late (2002-2010) periods: the Takashima Stroke Registry

\begin{tabular}{|c|c|c|c|c|c|}
\hline & \multicolumn{2}{|c|}{ 1990-2001 } & \multicolumn{2}{|c|}{ 2002-2010 } & $p$ value \\
\hline$N$ & 1,464 & & 907 & & \\
\hline Age, years & 71.9 & $(12.3)$ & 74.8 & $(12.1)$ & $<0.001$ \\
\hline Age 65 years and older, $N(\%)$ & 1,084 & (74.0) & 733 & $(80.8)$ & $<0.001$ \\
\hline Men, $N(\%)$ & 779 & $(53.2)$ & 490 & $(54.0)$ & 0.699 \\
\hline \multicolumn{6}{|l|}{ Stroke subtype, $N(\%)$} \\
\hline Cerebral infarction & 989 & $(67.6)$ & 608 & $(67.0)$ & \multirow[t]{4}{*}{0.009} \\
\hline Cerebral hemorrhage & 316 & $(21.6)$ & 227 & $(25.0)$ & \\
\hline Subarachnoid hemorrhage & 142 & $(9.7)$ & 70 & $(7.7)$ & \\
\hline Undermined stroke & 17 & $(1.2)$ & 2 & $(0.2)$ & \\
\hline \multicolumn{6}{|l|}{ Treatment, $N(\%)$} \\
\hline \multicolumn{6}{|l|}{ BP-lowering medication } \\
\hline No & 591 & $(53.3)$ & 406 & $(48.1)$ & \multirow[t]{3}{*}{0.022} \\
\hline Yes & 517 & $(46.7)$ & 438 & $(51.9)$ & \\
\hline Unknown & 356 & - & 63 & - & \\
\hline \multicolumn{6}{|l|}{ Glucose-lowering therapy } \\
\hline No & 827 & $(86.7)$ & 733 & $(85.8)$ & \multirow[t]{3}{*}{0.597} \\
\hline Yes & 127 & (13.3) & 121 & $(14.2)$ & \\
\hline Unknown & 510 & - & 53 & - & \\
\hline \multicolumn{6}{|l|}{ Lipid-lowering medication } \\
\hline No & - & - & 595 & $(91.5)$ & \multirow[t]{3}{*}{-} \\
\hline Yes & - & - & 55 & $(8.5)$ & \\
\hline Unknown & - & - & 257 & - & \\
\hline \multicolumn{6}{|l|}{ Smoking, $N(\%)$} \\
\hline Never smoker & 731 & $(66.6)$ & 478 & $(65.4)$ & \multirow[t]{4}{*}{$<0.001$} \\
\hline Past smoker & 13 & $(1.2)$ & 76 & (10.4) & \\
\hline Current smoker & 354 & $(32.2)$ & 177 & $(24.2)$ & \\
\hline Unknown & 366 & - & 176 & - & \\
\hline \multicolumn{6}{|l|}{ Drinking, $N(\%)$} \\
\hline Never drinker & 813 & $(73.8)$ & 486 & $(65.8)$ & \multirow[t]{4}{*}{$<0.001$} \\
\hline Past drinker & 10 & $(0.9)$ & 20 & $(2.7)$ & \\
\hline Current drinker & 279 & $(25.3)$ & 233 & $(31.5)$ & \\
\hline Unknown & 362 & - & 168 & - & \\
\hline History of TIA, N (\%) & 77 & (5.4) & 27 & $(2.5)$ & $<0.001$ \\
\hline \multicolumn{6}{|c|}{ Neuroimaging examination of index stroke, $N(\%)$} \\
\hline $\mathrm{CT}$ or MRI & 1,370 & $(93.6)$ & 871 & $(96.0)$ & 0.011 \\
\hline $\mathrm{CT}$ & 1,300 & (88.8) & 725 & (79.9) & $<0.001$ \\
\hline MRI & 651 & $(44.5)$ & 687 & (75.7) & $<0.001$ \\
\hline
\end{tabular}

Values are number, proportion (\%), or mean \pm standard deviation (\%). The $p$ values were calculated by the $t$ test for continuous variables and the $x^{2}$ test for categorical variables. All values categorized as "unknown" were excluded from the calculation. BP, blood pressure; $C T$, computed tomography; $M R I$, magnetic resonance imaging; $N$, number; TIA, transient ischemic attack.

od, while there were no significant differences in frequency of patients who received glucose-lowering therapies between the early and late periods. The smoking rate decreased from $32.2 \%$ in the early period and $24.2 \%$ in the late period.

Table 2 shows the age-standardized incidence rates of total stroke and its subtypes (per 100,000 person-years) in the early and the late periods. Age- and sex-standardized incidence rates using 2015 Japanese population of total stroke were 302 in the early and 191 in the late periods. In men, age-standardized incidence rates of total stroke were 330 in the early and 211 in the late periods. In women, agestandardized incidence rates of total stroke were 281 in the early and 172 in the late periods. Crude incidence rates of total stroke were 225 in the early and 187 in the late period (online suppl. Table 1; for all online suppl. material, see 
Table 2. Age- and sex-standardized incidence rates of first-ever stroke in the early (1990-2001) and late (2002-2010) periods: the Takashima Stroke Registry

\begin{tabular}{|c|c|c|c|c|}
\hline & \multicolumn{2}{|c|}{ 1990-2001 } & \multicolumn{2}{|c|}{$2002-2010$} \\
\hline & IR & $(95 \% \mathrm{Cl})$ & IR & $(95 \% \mathrm{Cl})$ \\
\hline \multicolumn{5}{|l|}{ Men and women } \\
\hline Total stroke & 302 & $(269,340)$ & 191 & $(165,223)$ \\
\hline Ischemic stroke & 209 & $(182,240)$ & 129 & $(108,155)$ \\
\hline Intracerebral hemorrhage & 62 & $(48,81)$ & 48 & $(36,66)$ \\
\hline Subarachnoid hemorrhage & 27 & $(18,41)$ & 14 & $(8,26)$ \\
\hline \multicolumn{5}{|l|}{ Men } \\
\hline Total stroke & 330 & $(281,389)$ & 211 & $(172,262)$ \\
\hline Ischemic stroke & 244 & $(204,295)$ & 156 & $(123,200)$ \\
\hline Intracerebral hemorrhage & 60 & $(41,89)$ & 45 & $(29,74)$ \\
\hline Subarachnoid hemorrhage & 21 & $(11,42)$ & 9 & $(4,26)$ \\
\hline \multicolumn{5}{|l|}{ Women } \\
\hline Total stroke & 281 & $(238,333)$ & 172 & $(141,215)$ \\
\hline Ischemic stroke & 181 & $(149,223)$ & 103 & $(80,137)$ \\
\hline Intracerebral hemorrhage & 64 & $(46,93)$ & 50 & $(35,76)$ \\
\hline Subarachnoid hemorrhage & 33 & $(20,55)$ & 19 & $(10,39)$ \\
\hline
\end{tabular}

Incidence rate indicates per 100,000 person-years. Age distribution was standardized to the 2015 Japanese population. Incidence rate for the unclassified stroke subtype was not presented due to a small number of patients. $\mathrm{Cl}$, confidence interval; IR, incidence rate.

Table 3. Average annual relative changes in incidence rates of first-ever stroke in the total 21-year period: the Takashima Stroke Registry (1990-2010)

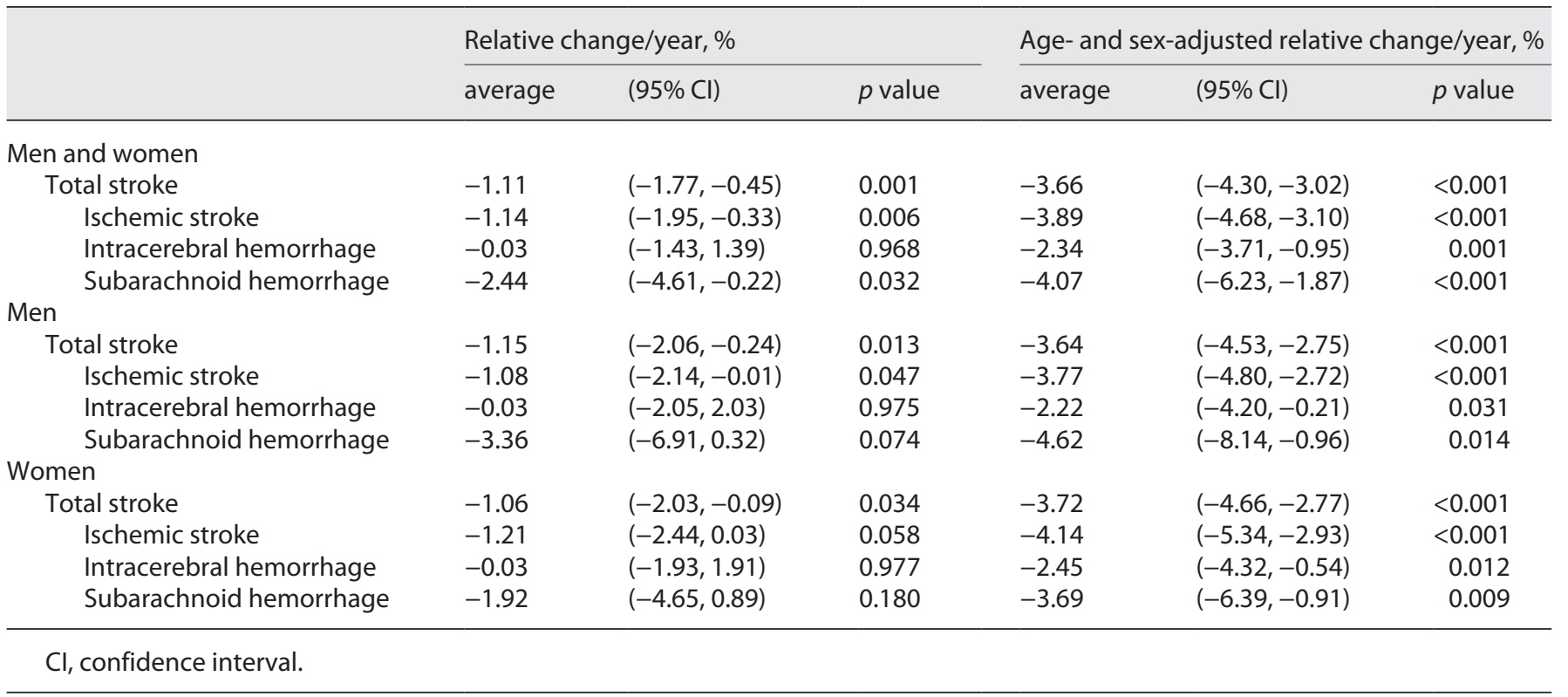

www.karger.com/doi/10.1159/000521643). Figure 1 shows trends in the crude and age-standardized incidence rates of first-ever total stroke and its subtypes. The trends in crude and age-standardized incidence rates of first-ever total stroke and its subtypes are shown separately for men and women in online supplementary Figures 1 and 2.

Trends in Stroke Incidence in Japan
Table 3 shows the crude and age- and sex-adjusted average annual relative change in stroke incidence during the total 21-year study period. A significantly decreasing trend of stroke incidence was observed in the crude analysis: average annual relative change in total stroke incidence was $-1.1 \%$ per year ( $95 \%$ CI: -1.8 to -0.5 ). A sig- 
Fig. 1. Trend in crude incidence rates of first-ever stroke (total stroke and its subtypes) (a) and age- and sex-adjusted incidence rates of first-ever stroke using 2015 Japanese population (b): the Takashima Stroke Registry (1990-2010).
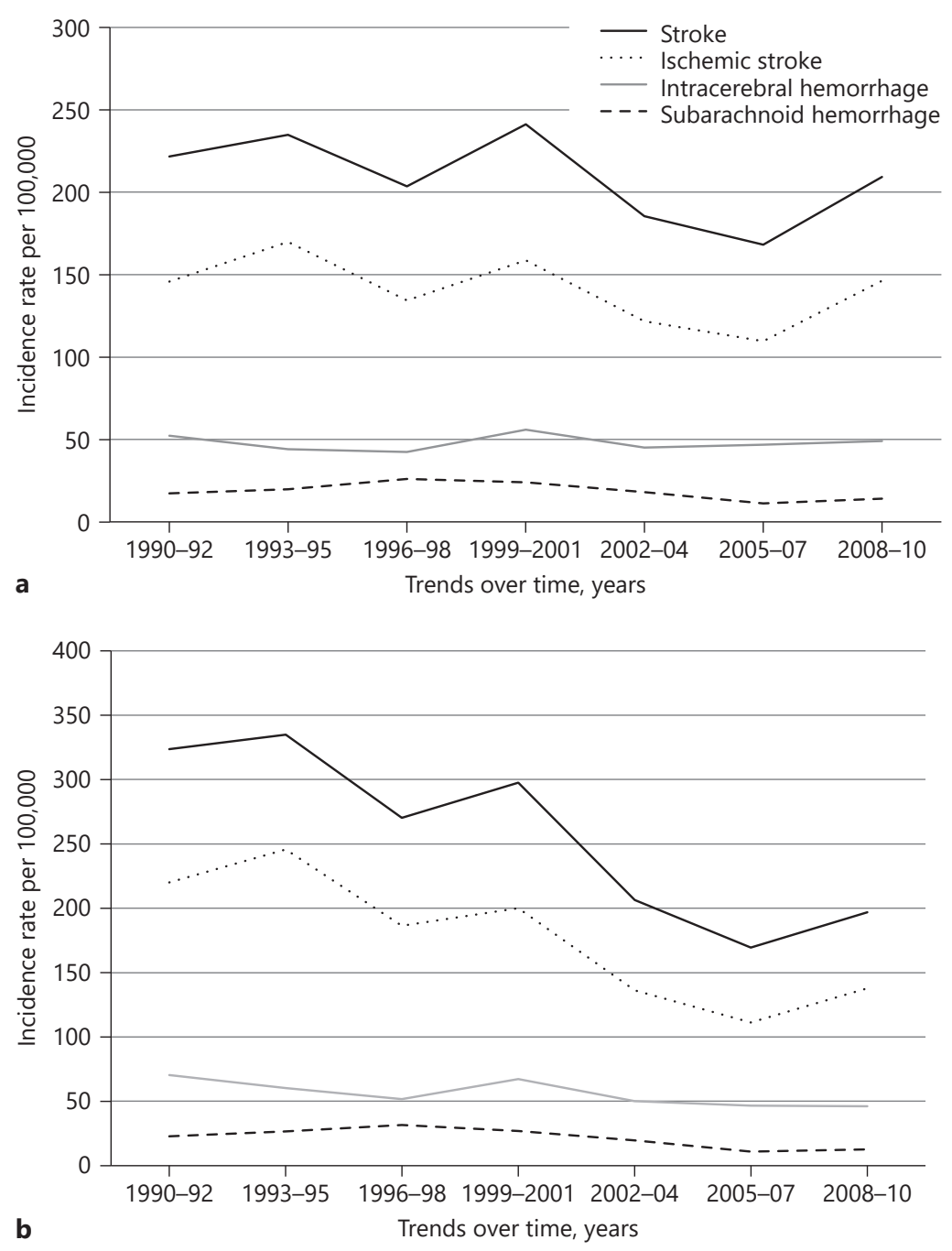

nificant annual relative reduction was also observed even after adjustment for age and sex: $-3.7 \%$ per year $(95 \% \mathrm{CI}$ : -4.3 to -3.0 ). Significant decreasing trends were observed in both in men and women, separately. Online supplementary Table 2 shows age- and sex-adjusted average annual relative changes in stroke incidence separately for the early (1990-2001) and late (2002-2010) study periods. During the early period, a significant decreasing trend in stroke incidence was observed ( $p$ for trend $=$ $0.006)$ with an annual percentage change of $-2.1 \%$ per year ( $95 \%$ CI: -3.5 to -0.6 ). During the late period, in contrast, there were no significant decreasing trends in incidence rates of total stroke $(p=0.60)$ with an annual percent change of $-0.7 \%$ per year (95\% CI: -3.1 to 1.9 ).

\section{Discussion}

In this long-term population-based stroke registry, we demonstrated significantly decreasing trends in incidence of total stroke and its subtypes over the 21-year study period. These reductions in stroke incidence remained statistically significant even after adjustment for age and sex. When the total study period was divided into early (19902001) and late (2002-2010) periods, a significant reduction in stroke incidence was observed only during the early period, while there were no significant reduction in incidence rates of stroke during the late period.

This is the first study to report the trend of stroke incidence in the 2000s based on the population-based stroke 
registry in Japan. In Japan, age-standardized stroke morality rates have continuously declined, and the rates were 98 in men and 69 in women in 1990, 74 in men and 46 in women in 2000, 50 in men and 27 in women in 2010, and 33 in men and 18 in women in 2019 [4, 13]. The decreasing time trend of stroke incidence observed in this study was similar to that of stroke mortality. However, continuous monitoring for the trend in stroke incidence is needed.

The Global Burden of Disease Study has reported reductions in the incidence of stroke from 1990 to 2016 in most regions of the world [1]. The estimation was consistent with our findings. A recent systematic review also reported that stroke incidence in the high-income European countries has declined steadily in the 21 st century [14]. However, the Dijon Stroke Registry showed increasing trend in incidence rates of ischemic stroke in young adults in France from 1985 to 2011 [15]. In Japan, the incidence and mortality of stroke declined dramatically from the 1960s to the 1990s [3, 5]. However, populationbased studies conducted in Sweden and Japan reported no clear changes in stroke incidence and mortality during the early 2000s [6, 14, 16, 17]. According to our sensitivity analysis, in the later period (2002-2010), the decreasing trends of stroke incidence might have slowed down. Further studies might be needed to observe the trend of stroke incidence during the 2010s.

The key risk factors for stroke in Japan are smoking $[18,19]$, hypertension $[17,19,20]$, and metabolic risk factors [21] including obesity [22], diabetes [23], and dyslipidemia [19]. During the 2000s, the overall smoking rate continued to decline [7]. Average BP levels declined during the 1990s [3], but declining trends in average BP levels slowed down during the 2000s [7]. In contrast, the prevalence of dyslipidemia continuously increased over this period $[4,7]$. These unfavorable trends in metabolic risk factors, which may be due to Westernization of lifestyle, are likely to be attributable to the observed slowdown in reduction of stroke incidence during the 2000s in Japan. Therefore, further countermeasures of these risk factors are required to provide a further reduction in stroke.

To our knowledge, this is the first study to report the trends in stroke incidence in the 2000s based on a comprehensive, population-based stroke registry in Japan. This study also has several limitations. First, our registry system did not cover clinics in Takashima City. However, in Japan, public health insurance systems under the control of the government covered almost $100 \%$ of residents. Therefore, a patient having stroke-like symptoms who visited general physicians in the area is almost always transferred to the hospitals in Takashima City. Moreover, 24-h emergency transport systems by local governments are available for all residents without charge, and the systems transport the patients to emergency hospitals. Therefore, it was almost no chance that someone having stroke which met the criteria of this study would be left out of our registry system. Second, the registry covers a rural area in Western Japan that may be different from the population in Eastern Japan where a higher mortality rate of stroke was observed. Furthermore, the use of magnetic resonance imaging scans became more widely used in the later years of this study, which may have influenced the diagnosis of minor stroke in the late period. However, we excluded all asymptomatic stroke cases according to the definition in the protocol. Therefore, the possibility of overdiagnosis is less likely to have affected our findings. Third, stroke cases with very minor symptoms who did not present to hospitals might be missed from our registry. Fourth, the denominator of the incidence rate in this study was person-years based on the total number of the city population (including prevalent stroke cases). This fact might have been resulted in underestimation of incidence rates in this study. However, this limitation has limited impact on the findings of this study because of low prevalence rates of stroke.

\section{Conclusions}

This population-based stroke registry in Japan observed a significant reduction in incidence rates of stroke from 1990 to 2010. These findings indicated the importance of continuous monitoring for the trend in stroke incidence. Facing super-aged society in Japan, these findings also re-emphasize the importance of public health measures to provide continuous protection against stroke.

\section{Acknowledgments}

We thank all investigators, participating hospitals, and study staff of the Takashima Study group. We thank John Daniel from Edanz Group (https://en-author-services.edanz.com/ac) for language polishing a draft of this manuscript.

\section{Statement of Ethics}

The Takashima Stroke Registry protocol was approved by the Institutional Review Board of the Shiga University of Medical Science (R2000-014), and the President of the Shiga University of Medical Science gave the waiver for written informed consent. 


\section{Conflict of Interest Statement}

Okayama A. is the President of the Research Institute of Strategy for Prevention. Arima H. is an associate editor of Cerebrovascular Diseases. The other authors declare that there are no conflicts of interest.

\section{Funding Sources}

This work was supported by the Research on Cardiovascular Disease (3A-1, 6A-5, 7A-2), the Comprehensive Research on Cardiovascular and Life Style Related Disease (H18-CVD-Ippan-029) of the Ministry of Health, Labour and Welfare, and Grants-in-Aid Scientific Research (19H03902, 24390165, B17390186, B20390184, C213670361, and 17015018) of the Ministry of Education, Culture, Sports, Science and Technology, Japan.

\section{Author Contributions}

Kita Y., Okayama A., and Ueshima H. contributed to the conception of this study. Takashima N., Kita Y., Turin T.C., Okayama A., Miura K., and Ueshima H. contributed to the design of this study. Takashima N., Turin T.C., Nakamura Y., Sugihara H., Morita Y., Okayama A., Ueshima H., and Kita Y. contributed to the acquisition of data. Takashima N. and Arima H. contributed to the analysis of this study and contributed to the interpretation of data. Takashima N. drafted the manuscript for the work, and Arima H. supervised the writing of the manuscript. All authors revised the manuscript, approved the manuscript to be published, and agree to be accountable for all aspects of the work in ensuring that questions related to the accuracy or integrity of any part of the work are appropriately investigated and resolved.

\section{Data Availability Statement}

The data that support the findings of this study cannot be shared due to ethical restrictions. Queries regarding the data in this article should be addressed to the corresponding author (N.T.).

\section{References}

1 Global Burden 2016 Stroke Collaborators. Global, regional, and national burden of stroke, 1990-2016: a systematic analysis for the global burden of disease study 2016. Lancet Neurol. 2019;18:439-58.

2 Liu L, Ikeda K, Yamori Y. Changes in stroke mortality rates for 1950 to 1997 : a great slowdown of decline trend in Japan. Stroke. 2001; 32:1745-9.

3 Ueshima H. Explanation for the Japanese paradox: prevention of increase in coronary heart disease and reduction in stroke. J Atheroscler Thromb. 2007;14:278-86.

4 E-Statistic. Tokyo, Japan: statistics bureau, ministry of internal affairs and Communications. Japan; 2021.

5 Hata J, Ninomiya T, Hirakawa Y, Nagata M, Mukai N, Gotoh S, et al. Secular trends in cardiovascular disease and its risk factors in Japanese: half-century data from the Hisayama Study (1961-2009). Circulation. 2013;128: 1198-205.

6 Hisamatsu T, Segawa H, Kadota A, Ohkubo T, Arima H, Miura K. Epidemiology of hypertension in Japan: beyond the new 2019 Japanese guidelines. Hypertens Res. 2020;43: 1344-51.

7 Ministry of Health LaW. The national health and nutrition survey in Japan. Office of nutrition HSD, health service bureau, ministry of health, labour and welfare. Tokyo, Japan: Ministry of Health, Labour and Welfare; 2018.

8 Ikeda N, Nishi N, Noda H, Noda M. Trends in prevalence and management of diabetes and related vascular risks in Japanese adults: Japan national health and nutrition surveys 2003-2012. Diabetes Res Clin Pract. 2017; 127:115-22.
9 Kita Y, Okayama A, Ueshima H, Wada M, Nozaki A, Choudhury SR, et al. Stroke incidence and case fatality in Shiga, Japan 19891993. Int J Epidemiol. 1999;28:1059-65.

10 Kita Y, Turin TC, Rumana N, Sugihara H, Morita Y, Hirose K, et al. Surveillance and measuring trends of stroke in Japan: the Takashima Stroke Registry (1988 - present). Int J Stroke. 2007;2:129-32.

11 Kita Y, Turin TC, Ichikawa M, Sugihara H, Morita Y, Tomioka N, et al. Trend of stroke incidence in a Japanese population: Takashima stroke registry, 1990-2001. Int J Stroke. 2009;4:241-9.

12 Tunstall-Pedoe H. Monitoring trends in cardiovascular disease and risk factors: the WHO "Monica" project. WHO Chron. 1985;39:3-5.

13 Shiga prefecture. Shiga prefecture statistical report. Shiga, Japan: Shiga prefecture; 2020.

14 Li L, Scott CA, Rothwell PM. Trends in stroke incidence in high-income countries in the 21st century: population-based study and systematic review. Stroke. 2020;51:1372-80.

15 Bejot Y, Daubail B, Jacquin A, Durier J, Osseby $\mathrm{GV}$, Rouaud O, et al. Trends in the incidence of ischaemic stroke in young adults between 1985 and 2011: the Dijon stroke registry. J Neurol Neurosurg Psychiatry. 2014;85:509-13.

16 Harmsen P, Wilhelmsen L, Jacobsson A. Stroke incidence and mortality rates 1987 to 2006 related to secular trends of cardiovascular risk factors in Gothenburg, Sweden. Stroke. 2009;40:2691-7.

17 Takashima N, Ohkubo T, Miura K, Okamura T, Murakami Y, Fujiyoshi A, et al. Long-term risk of $\mathrm{BP}$ values above normal for cardiovascular mortality: a 24-year observation of Japanese aged 30 to 92 years. J Hypertens. 2012;30: 2299-306.
18 Ueshima H, Choudhury SR, Okayama A, Hayakawa T, Kita Y, Kadowaki T, et al. Cigarette smoking as a risk factor for stroke death in Japan: NIPPON DATA80. Stroke. 2004;35: 1836-41.

19 NIPPON DATA 80 Research Group. Risk assessment chart for death from cardiovascular disease based on a 19-year follow-up study of a Japanese representative population. Circ J. 2006;70:1249-55.

20 NIPPON DATA 80 Research Group. Impact of elevated blood pressure on mortality from all causes, cardiovascular diseases, heart disease and stroke among Japanese: 14 year follow-up of randomly selected population from Japanese - Nippon data 80. J Hum Hypertens. 2003; 17:851-7.

21 Kadota A, Hozawa A, Okamura T, Kadowaki T, Nakmaura K, Murakami Y, et al. Relationship between metabolic risk factor clustering and cardiovascular mortality stratified by high blood glucose and obesity: NIPPON DATA90, 1990-2000. Diabetes Care. 2007; 30:1533-8.

22 Yatsuya $\mathrm{H}$, Toyoshima $\mathrm{H}$, Yamagishi $\mathrm{K}$, Tamakoshi K, Taguri M, Harada A, et al. Body mass index and risk of stroke and myocardial infarction in a relatively lean population: meta-analysis of 16 Japanese cohorts using individual data. Circ Cardiovasc Qual Outcomes. 2010;3:498-505.

23 Hirakawa $\mathrm{Y}$, Ninomiya $\mathrm{T}$, Kiyohara $\mathrm{Y}, \mathrm{Mu}-$ rakami Y, Saitoh S, Nakagawa $\mathrm{H}$, et al. Agespecific impact of diabetes mellitus on the risk of cardiovascular mortality: an overview from the evidence for cardiovascular prevention from observational cohorts in the Japan research group (EPOCH-JAPAN). J Epidemiol. 2017;27:123-9. 